\title{
Heat shock protein 70 prevents secretagogue-induced cell injury in the pancreas by preventing intracellular trypsinogen activation
}

\author{
Lakshmi Bhagat, ${ }^{1}$ Vijay P. Singh, ${ }^{1}$ Antti J. Hietaranta, ${ }^{1}$ Sudhir Agrawal, ${ }^{2}$ \\ Michael L. Steer, ${ }^{1}$ and Ashok K. Saluja ${ }^{1}$ \\ ${ }^{1}$ Department of Surgery, Beth Israel Deaconess Medical Center and Harvard Medical School, Boston, Massachusetts, USA \\ ${ }^{2}$ Hybridon Inc., Milford, Massachusetts, USA \\ Address correspondence to: Ashok K. Saluja, DA 817, Department of Surgery, Beth Israel Deaconess Medical Center, \\ 330 Brookline Avenue, Boston, Massachusetts 02215, USA. Phone: (617) 667-5369; Fax: (617) 667-8679; \\ E-mail: asaluja@bidmc.harvard.edu.
}

Received for publication October 18, 1999, and accepted in revised form May 31, 2000.

Rodents given a supramaximally stimulating dose of cholecystokinin or its analogue cerulein develop acute pancreatitis with acinar cell injury, pancreatic inflammation, and intrapancreatic digestive enzyme (i.e., trypsinogen) activation. Prior thermal stress is associated with heat shock protein 70 (HSP70) expression and protection against cerulein-induced pancreatitis. However, thermal stress can also induce expression of other HSPs. The current studies were performed using an in vitro system to determine whether HSP70 can actually mediate protection against pancreatitis and, if so, to define the mechanism underlying that protection. We show that in vitro exposure of freshly prepared rat pancreas fragments to a supramaximally stimulating dose of cerulein results in changes similar to those noted in cerulein-induced pancreatitis, i.e., intra-acinar cell trypsinogen activation and acinar cell injury. Short-term culture of the fragments results in HSP70 expression and loss of the pancreatitis-like changes noted after addition of cerulein. The culture-induced enhanced HSP70 expression can be prevented by addition of either the flavonoid antioxidant quercetin or an antisense oligonucleotide to HSP70. Under these latter conditions, addition of a supramaximally stimulating concentration of cerulein results in trypsinogen activation and acinar cell injury. These findings indicate that the protection against cerulein-induced pancreatitis that follows culture-induced (and possibly thermal) stress is mediated by HSP70. They suggest that the HSP acts by preventing trypsinogen activation within acinar cells.

J. Clin. Invest. 106:81-89 (2000).

\section{Introduction}

Rats infused with a supramaximally stimulating dose of cerulein, a cholecystokinin analogue, develop acute interstitial pancreatitis (1). We have recently shown that the early events that characterize secretagogueinduced pancreatitis can also be induced by the in vitro exposure of rat pancreatic acini to a supramaximally stimulating concentration of cerulein $(2,3)$. Under both in vivo and in vitro conditions, one of the earliest events that follows supramaximal secretagogue stimulation is the intra-acinar cell activation of digestive zymogens such as trypsinogen $(3,4)$. This intracellular activation of digestive enzymes subsequently leads to pancreatic injury in vivo and to acinar cell injury in vitro (5). We have suggested that this in vitro reductionist model system of pancreatitis might prove useful for studies directed at identifying cellular events that regulate the evolution and severity of pancreatitis.

Exposure of rats to thermal stress, 12 to 24 hours before cerulein infusion, prevents secretagogue-induced pancreatitis (6). Whereas this prior period of thermal stress is known to induce heat shock protein 70 (HSP70 [inducible]; also known as HSP72) expression in the pancreas, the levels of other HSPs, including HSP27, are also upregulated following thermal stress (ref. 7 and A.K. Saluja et al., unpublished observations). Also, other potentially protective changes may follow thermal stress and, for that reason, it has not been possible to conclude that the protection against secretagogue-induced pancreatitis that follows thermal stress is actually mediated by upregulated HSP70 expression. In addition, the mechanisms responsible for HSP70-mediated protection, if it were to occur, remain unknown.

In this study, we have used an in vitro system of cultured pancreas fragments to explore the relationships between HSP70 expression and secretagogue-induced acinar cell injury. As had been noted for freshly isolated acini (5), we find that exposure of freshly prepared fragments to a supramaximally stimulating concentration of cerulein leads to colocalization of digestive enzymes and lysosomal hydrolases, trypsinogen activation, and, subsequently, to acinar cell injury. Short-term culture of freshly prepared fragments does not alter their secretory response to cerulein but, under these conditions, supramaximal stimulation no longer causes colocalization, trypsinogen activation, or cell injury. Short-term 
culture of fragments also induces HSP70 expression in these fragments. Prevention of upregulated HSP70 expression, either by incubation with the flavonoid antioxidant quercetin or by incorporation of an antisense HSP70 oligonucleotide, prevents HSP70 upregulation and restores all of those pancreatitis-like responses (i.e., colocalization, trypsinogen activation, and cell injury) to supramaximal cerulein stimulation. These observations lead us to conclude that the upregulated expression of HSP70 can prevent secretagogue-induced acinar cell injury and that the HSP exerts this protective effect by preventing trypsinogen activation.

\section{Methods}

All experimental protocols were approved by the Institutional Animal Care and Use Committee of the Beth Israel Deaconess Medical Center, Boston, Massachusetts, USA. Cerulein, the decapeptide analogue of the potent pancreatic secretagogue cholecystokinin, was purchased from Research Plus Inc. (Bayonne, New Jersey, USA); trypsin substrate (BoC-Glu-Ala-Arg-MCA) from Peptides International (Louisville, Kentucky, USA); HSP70/HSP72 (SPA-810), HSC70/HSP73 (SPA815), HSP60 (SPA-806), HSP25/27 (SPA-801), and anti-rat IgG-HRP-conjugated antibodies from StressGen Biotechnologies Corp. (Victoria, British Columbia, Canada); and anti-cathepsin B, anti-mouse and antirabbit IgG-HRP conjugates, anti-rabbit IgG-FITC, and anti-goat IgG-rhodamine conjugates from Santa Cruz Biotechnology Inc. (Santa Cruz, California, USA). Antirabbit trypsinogen-activation peptide (TAP) antibody was a kind gift from D. McNally, Abbott Laboratories (Abbott Park, Illinois, USA). The reagent 3-[4,5dimethylthiazol-2-yl]-2,5-diphenyltetrazolium bromide (MTT) was from Molecular Probes Inc. (Eugene, Oregon, USA); DAPI/antifade was from Oncor (Gaithersburg, Maryland, USA); Superfrost/Plus slides from Fisher Scientific (Pittsburgh, Pennsylvania, USA); F-12K Nutrient Mixture (Kaighn's modification), penicillin-streptomycin, Superscript II reverse transcriptase, and PCR primers were from GIBCO BRL (Grand Island, New York, USA). FBS was purchased from Atlanta Biologicals (Norcross, Georgia, USA); RNA Later and Totally RNA Isolation kit was from Ambion (Austin, Texas, USA); Ready Gels (10\% SDS) and nitrocellulose membranes from BioRad Laboratories (Hercules, California, USA); and Renaissance Western Blot Chemiluminescence Reagent Plus from NEN Life Science Products (Boston, Massachusetts, USA). All other chemicals and reagents were purchased from Sigma Chemical Co. (St. Louis, Missouri, USA).

Antisense and sense oligonucleotides. Two antisense (AS I and AS II), and their corresponding sense (S I and S II), oligonucleotides, corresponding to different regions of the HSP70 gene, were used in the present study. All oligonucleotides were phosphorothioated and synthesized by Hybridon Inc. (Milford, Massachusetts, USA). A 14-base phosphorothioate AS nucleotide (5'-CAGGTCGATGCCGA-3'), referred to as AS I, was synthesized to a highly conserved region of HSP70 (inducible form) as described in detail by Nakano et al. (8). The sequence 5'TCGGCATCGACCTG-3' (sense, S) corresponds to bases 508 to 521 in the human gene (9) (Genbank accession no. M11717 M15432; National Institutes of Health [NIH], Bethesda, Maryland, USA) and occurs very close to the translation start site at base 489. A search of the $\mathrm{NIH}$ Genbank for mammalian genes with the same sequence identified 18 different exact matches, all HSP70 genes from various species, including human, cow, pig, rat, hamster, and mouse. A comparison of this sequence for human HSP70 with the human HSC70 (heat shock cognate form) showed that five out of 14 bases did not match (TTGGTATTGATCTT; Genbank accession no. Y00371) (10). Thus, there is significant sequence difference between HSC70 and HSP70 in this 14-base region. The second 18-base phosphorothioate antisense oligonucleotide (AS II) was synthesized on the basis of the coding region (5'-CACCTTGCCGTGCTGGAA$3^{\prime}$, nucleotide positions 61-78) of the mouse HSP70 gene (11) as described by Robertson et al. (12). In this case, also, no significant sequence similarity was found to either the HSC70 or GRP 78 genes. (GRP 78 is a member of HSP 70 family of proteins, also referred to as $\mathrm{BiP}$, which is primarily localized in the endoplasmic reticulum.) Sense phosphorothioate nucleotides to both these AS nucleotides were used as controls.

Preparation and culture of pancreas fragments and evaluation of viability. Pancreas fragments were prepared essentially according to the method of Jaffrey et al. (13). Pancreata from Wistar rats (75-100 gm) were removed and injected with HEPES buffer, $\mathrm{pH} 7.4$, containing $\mathrm{NaCl}$ (130 mM), $\mathrm{KCl}(5 \mathrm{mM}), \mathrm{MgCl}_{2}(1 \mathrm{mM}), \mathrm{CaCl}_{2}(1 \mathrm{mM})$, HEPES $(10 \mathrm{mM})$, glucose $(15 \mathrm{mM})$, sodium pyruvate $(10 \mathrm{mM})$, and BSA $(0.1 \%)$. The buffer was saturated with oxygen by bubbling. The fragments were then cut into small pieces less than $0.5 \mathrm{~mm}$ in diameter, resuspended in Ham's F12K nutrient mixture supplemented with $20 \%$ FBS, and cultured in 24-well plates for up to 48 hours. At different times the fragments were harvested, washed with HEPES buffer, and their response to cerulein administration was studied.

The viability of pancreas fragments was determined using MTT, as described by Jaffrey et al. (13).

Treatment of fragments with quercetin and antisense oligonucleotides. A fresh stock of quercetin was prepared in DMSO and added to the medium (50 $\mu \mathrm{M}$ final concentration) at the time the fragments were placed in wells. Rat pancreas fragments were also cultured either with phosphorothioate AS (AS I or AS II,1 $1 \mu \mathrm{M}$ ) to HSP70 or $1 \mu \mathrm{M}$ sense (S I or S II) RNA dissolved in distilled water and added to the culture medium. Fragments were incubated with these agents for a period of 12 hours, then washed thoroughly, and used for further experiments. Fragments incubated in the medium only served as controls. In preliminary experiments, four concentrations of AS nucleotides $(0.5,1,2$, and $4 \mu \mathrm{M})$ were tested. Since incubation with $1 \mu \mathrm{M}$ AS I or AS II for 12 hours was sufficient to inhibit HSP70 expression and 
also to result in restoration of trypsinogen activation in response to supramaximal dose of cerulein, this dose of AS was chosen for all further experiments.

Also, preliminary studies were done with FITC endlabeled AS II nucleotide to examine its uptake by pancreas fragments for a period of up to 18 hours in culture. Freshly prepared pancreas fragments were incubated with labeled AS (100 nM), and 12 and 18 hours later samples were harvested, washed thoroughly with PBS, homogenized, and the fluorescent intensity measured (excitation $494 \mathrm{~nm}$; emission $520 \mathrm{~nm}$ ) and expressed as fluorescent intensity/total protein. Since there was no increase in uptake after 12 hours $(463.4 \pm 30.2,12$ hours, vs. $439.1 \pm 29.4$, 18 hours), this period of incubation was chosen for all treatments. Morphological examination of pancreas fragments under a fluorescence microscope confirmed the uptake of AS nucleotide as green fluorescent punctate dots in the acinar cells.

Western blotting for HSPs. Pancreas fragments were washed thoroughly with HEPES buffer after various treatments and then homogenized in suspension buffer, $\mathrm{pH} 7.4$, containing $\mathrm{NaCl}(100 \mathrm{mM})$, Tris $\mathrm{HCl}$ $(10 \mathrm{mM})$, EDTA $(1 \mathrm{mM})$, aprotinin $(1 \mu \mathrm{g} / \mathrm{mL})$, and PMSF $(100 \mu \mathrm{g} / \mathrm{mL})$. The homogenates were centrifuged $\left(14,000 \mathrm{~g}\right.$ for 15 minutes at $\left.4{ }^{\circ} \mathrm{C}\right)$, the supernatants collected, and the protein concentration in the supernatant was measured by the method of Bradford (14). Samples $(10 \mu \mathrm{g} / \mathrm{lane})$ were subjected to gel electrophoresis as described by Laemmli (15) using $10 \%$ Ready gels and transferred onto nitrocellulose membranes for Western blot analysis by the method of Towbin et al. (16). Induction of HSP27, HSP60, HSC70, and HSP70 was monitored using antibodies to these HSPs $(1: 1,000)$. Labeled proteins were visualized by the enhanced chemiluminescence method using HRP-coupled secondary antibodies $(1: 5,000)$ and quantitated using a Scion Image Analysis Program (Scion Corp., Frederick, Maryland, USA).

RNA isolation and RT-PCR for HSP70 and HSP60. Pancreas fragments were washed with HEPES buffer and stored in RNA Later at $4^{\circ} \mathrm{C}$ until further processing. Total RNA was extracted using the Totally RNA kit. Five micrograms of total RNA was reverse transcribed in a 50$\mu \mathrm{L}$ reaction mixture containing $50 \mathrm{ng}$ of random hexamer as primer, together with 200 U Superscript II reverse transcriptase, according to the manufacturer's instructions (GIBCO BRL). For the PCR, $2 \mu \mathrm{L}$ of cDNA mixture were added to $50 \mu \mathrm{L}$ of a master mix containing $200 \mu \mathrm{M}$ of each dNTP, $100 \mathrm{pM}$ of each specific primer, as well as $1.5 \mathrm{mM} \mathrm{MgCl}_{2}$ and $25 \mathrm{U}$ Taq DNA polymerase. DNA oligonucleotide primers for HSP70 were selected from NIH Genbank (accession no. E12384). The sense primer was based on the sequence number 631-652 5'-GCTGACCAAGATGAAGGAGATC- $3^{\prime}$ and the antisense number 1100-1019 5'-GAGTCGATCTCCAGGCTGGC-3'. The sequences for HSP60 were also selected from NIH Genbank (accession no. X53584). The sequence for sense HSP60 was 5'-TAGGGAAAGTTGGAGAGGTC-3' and antisense 5'-AGTTAGAACATGCCGCCTCC-3'. The optimal reaction conditions chosen were an annealing temperature of $40^{\circ} \mathrm{C}$, cycle number 35 , and $\mathrm{Mg}^{2+}$ concentration of $1.5 \mathrm{mM}$. The PCR products were separated on $1.5 \%$ agarose gel and stained with ethidium bromide. The stained gels were photographed under ultraviolet (UV) illumination using Polaroid 667 film (Polaroid Corp., Cambridge, Massachusetts, USA).

Amylase secretion from pancreas fragments. Pancreas fragments, harvested at various time points, were incubated at $37^{\circ} \mathrm{C}$ with varying concentrations of cerulein for 30 minutes. Amylase secretion into the medium and total amylase content were measured using 4,6-ethylidene $\left(\mathrm{G}_{7}\right)$ - $p$-nitrophenyl $\left(\mathrm{G}_{1}\right)-\alpha_{1} \mathrm{D}$-maltoheptoside as the substrate, as described by Kruse-Jarres et al. (17). Amylase secretion into the medium was expressed as a percent of total amylase content.
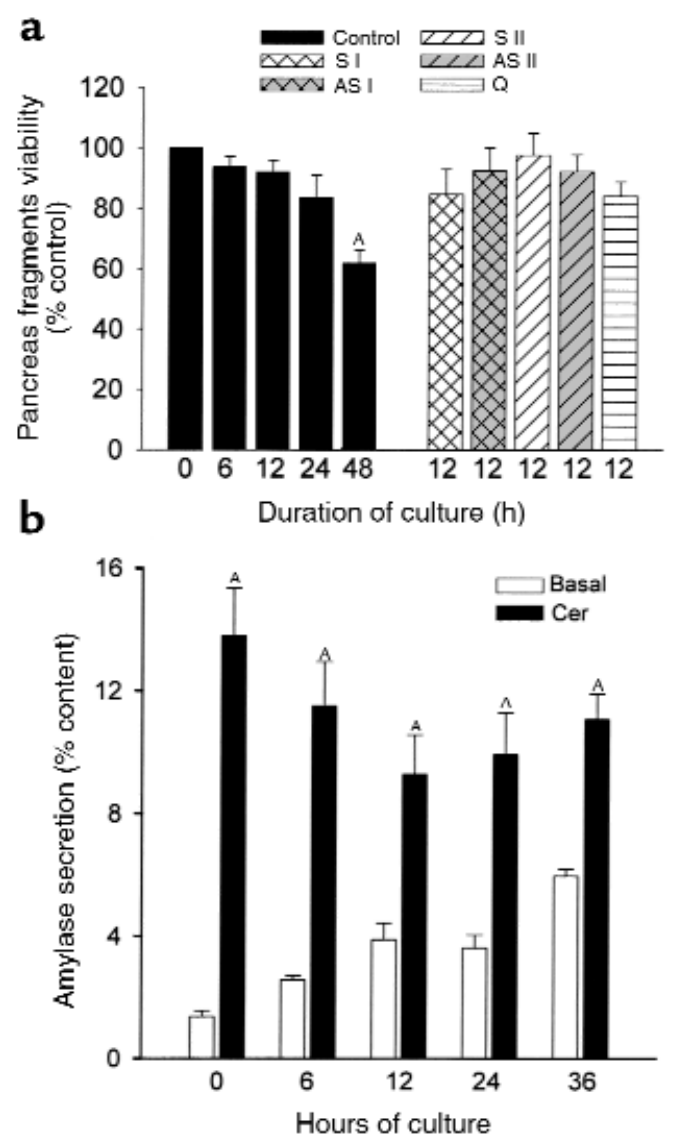

Figure1

Effect of duration of culture and incubation with oligonucleotides on the viability of pancreas fragments as assessed by MTT assay and amylase secretion. (a) MTT assay. S I and S II represent sense oligonucleotides, AS I and AS II represent antisense nucleotides, and $\mathrm{Q}$ represents quercetin as described in the text. Values are mean \pm SEM obtained from at least three separate experiments and expressed as percent of control ( 0 hours) values. ${ }^{A} P<0.05$ compared with freshly prepared (0 hours) samples. (b) Amylase release into the medium in response to $0.1 \mathrm{nM}$ cerulein was quantitated as described in the text. Values are mean \pm SEM for at least three separate experiments, each consisting of three or more wells in each group. AP $<0.05$ when cerulein-stimulated amylase secretion is compared with basal values at each time point indicated. 


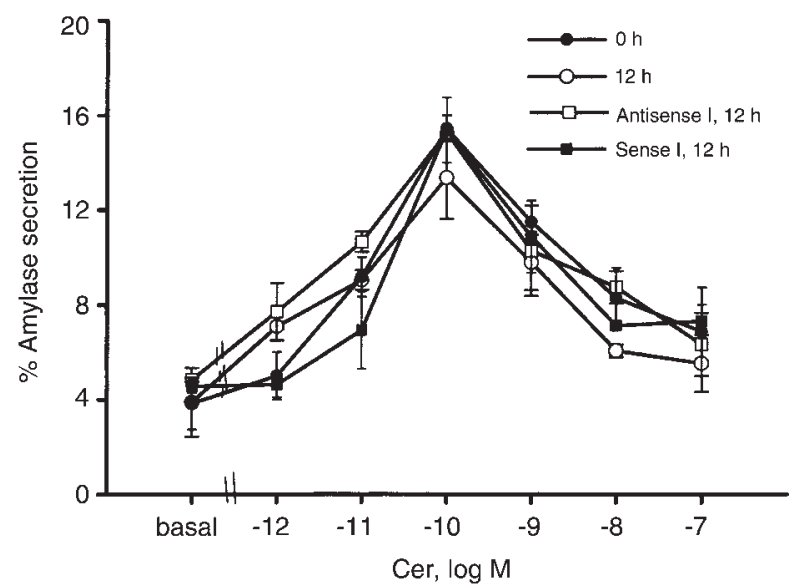

Figure 2

Effect of duration of culture and incubation with oligonucleotides on dose response for cerulein-stimulated amylase secretion from pancreas fragments. Amylase release into the medium was quantitated as described in the text. Values are mean \pm SEM for at least three separate experiments at each cerulein concentration in each group. Cer, cerulein.

Trypsin activity in pancreas fragments. After incubation of the pancreas fragments with or without cerulein for 30 minutes, the suspending buffer was removed and the fragments were washed twice with cold $\left(4^{\circ} \mathrm{C}\right)$ Mops buffer, pH 7.0 (250 $\mathrm{mM}$ sucrose, $5 \mathrm{mM}$ Mops, and 1 $\mathrm{mMMgSO}_{4}$ ). They were then homogenized in the same buffer using a motorized glass-Teflon homogenizer. The resulting homogenate was centrifuged $(50 \mathrm{~g}, 5 \mathrm{~min}$ utes), and the supernatant was taken for assay. Trypsin activity was measured fluorometrically using BoC-GluAla-Arg-MCA as the substrate, according to the method of Kawabata et al. (18) and described in detail in our earlier publication (4). DNA was measured using Hoechst dye 33258, according to the method of Labarca and Paigen (19) and the results expressed as trypsin activity (slope) per microgram of DNA in the homogenate.

Trypsinogen activation peptide levels in pancreas fragments. Immediately after treatment of the fragments with 0.1 $\mu \mathrm{M}$ cerulein for 30 minutes, the suspending medium was removed, the fragments were resuspended in trypsinogen activation peptide (TAP) assay buffer, $\mathrm{pH}$ 7.4 (20 mM Tris-HCl, 5 mM EDTA, $150 \mathrm{mM} \mathrm{NaCl}$ ), and the samples were boiled for 10 minutes. They were then homogenized in the same buffer, centrifuged, and the supernatant was stored frozen for later TAP measurement. TAP levels were determined by direct competitive ELISA using affinity-purified rabbit polyclonal antibody to TAP, according to the method of Gudgeon et al. (20). TAP levels were expressed as nanograms of TAP per microgram of DNA.

Light microscopy immunolocalization of TAP and cathepsin $B$. Fragments (freshly prepared or after 12 hours of culture with or without antisense/sense oligonucleotides) after 30 minutes of stimulation with $0.1 \mu \mathrm{M}$ cerulein were fixed in neutral buffered formalin and, after processing, were embedded in paraffin wax. Immunolocal- ization of TAP and cathepsin B was carried out using the protocol described by Hofbauer et al. (4). Photomicrographs were taken using BioRad MRC1200ES confocal laser scanning microscope, with the fluorescence in two channels monitored simultaneously. The fluorescence attributable to TAP appears green, whereas that attributable to cathepsin B appears as red. Thus, areas of colocalization appear as yellow dots in photomicrographs.

Lactic dehydrogenase release from pancreas fragments. Pancreas fragments were incubated for 90 minutes with 0.1 $\mu \mathrm{M}$ cerulein, and the percent of total lactic dehydrogenase (LDH) activity released into the suspending medium was quantitated. LDH activity was assayed as described by Amador et al. (21).

Statistical analysis. The results reported represent mean plus or minus SEM values obtained from multiple determinations in three or more separate experiments. The significance of changes was evaluated using student's $t$ test when the data consisted of only two groups or by ANOVA when comparing three or more groups. A $P$ value of less than 0.05 was considered to be statistically significant.

\section{Results}

Viability and functional integrity of pancreas fragments. MTT penetrates cells, and viable cells with active mitochondria cleave the tetrazolium ring into a dark blue formazan reaction product, which can be quantitated spectrophotometrically. We used MTT uptake and cleavage as a measure of viability for the pancreatic fragments. As shown in Figure 1a, fragments maintain their viability for up to 48 hours in culture. After 6-24 hours of culture, viability is similar to that of freshly prepared fragments, but it decreases over the subse-

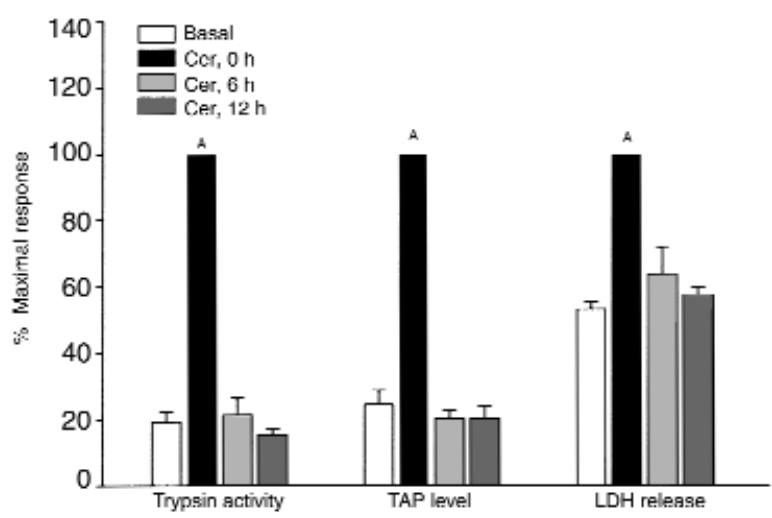

\section{Figure 3}

Effect of duration of culture on the ability of supramaximal cerulein to cause trypsinogen activation (as assessed by measuring trypsin activity and TAP levels) and cell injury (LDH leakage) in pancreas fragments. Values are mean \pm SEM obtained from at least three independent experiments and expressed as percent of maximal response obtained when freshly prepared pancreas fragments are stimulated with supramaximal dose of cerulein. ${ }^{A} P<0.05$ when values obtained from cerulein-stimulated freshly prepared fragments are compared with the basal values. 


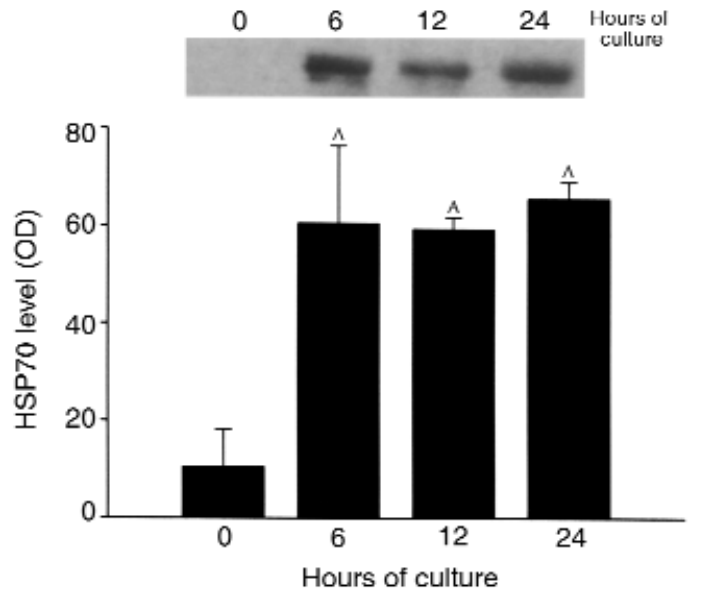

Figure 4

Effect of duration of culture on HSP70 expression in pancreas fragments, as assessed by Western blotting. Relative optical densities were quantitated as described in the text and are expressed as mean \pm SEM for at least three independent experiments in each group. A $P<0.05$ compared with control ( 0 hour) value.

quent 24 hours. Inclusion of quercetin, antisense (AS I, AS II), or sense (S I, S II) oligonucleotides in the culture medium also does not affect the viability of fragments.

Cerulein, an analogue of cholecystokinin, induces biphasic secretion of amylase from pancreas fragments with stimulation of secretion in the presence of low concentrations of cerulein and inhibition at higher concentrations of the secretagogue. We used cerulein stimulation/inhibition of amylase secretion as a measure of the functional integrity of pancreas fragments. As shown in Figure $1 \mathrm{~b}$ and 2, pancreas fragments remain responsive to cerulein for up to 36 hours. There is a gradual, time-dependent rise in basal amylase secretion from the fragments as well as a gradual decline in the maximal rate of amylase secretion noted with 0.1 $\mathrm{nM}$ cerulein, but net stimulation of secretion persists (Figure 1b). That stimulation retains its biphasic character when varying concentrations of cerulein are used, whether the fragments are freshly prepared or cultured for 12 hours in the absence or presence of sense (S I) or antisense (AS I) oligonucleotides (Figure 2). Similar results were obtained with S II and AS II oligonucleotides (data not shown).

Effects of culture on cerulein-induced trypsinogen activation and cell injury. We have recently shown that incubation of freshly prepared pancreatic acini with a supramaximally stimulating concentration of cerulein results in intra-acinar cell activation of trypsinogen, release of TAP within acinar cells, and acinar cell injury $(4,5)$. As shown in Figure 3, the same response to a supramaximally stimulating concentration of cerulein is noted when freshly prepared fragments are used. When fragments are cultured for 6 or 12 hours, basal levels of trypsin activity, TAP, and LDH release are similar to those noted for freshly prepared fragments (not shown). Addition of a supramaximally stimulating concentration of cerulein to fragments after either 6 or 12 hours of culture, however, fails to result in a rise in trypsin activity or TAP levels, and LDH leakage is not accelerated (Figure 3).

Effects of culture on HSP levels and message in pancreas fragments. HSP70 levels, as determined by Western blotting, are relatively low in freshly prepared fragments, but they rise within 6 hours after the start of culture and remain stably elevated for up to 24 hours of culture (Figure 4). HSC70, HSP27 (Figure 5b), and HSP60 (data not shown) levels were not altered by the culture of fragments over this time. HSP70 mRNA levels, as determined by RT-PCR, are also low in freshly prepared fragments, and they also rise rapidly within 6 hours after the start of culture (data not shown).

Effects of quercetin and antisense oligonucleotides on HSP70 expression in pancreas fragments. Incubation of freshly prepared fragments with the antioxidant quercetin (50 $\mu \mathrm{M})$ for 12 hours was found to completely block the otherwise expected rise in HSP70 levels (Figure 5a), and the resulting HSP70 level was noted to be below that observed in freshly prepared fragments at the start of incubation. A similar result was obtained when freshly prepared fragments were incubated with phosphorothioate antisense nucleotide (AS I or AS II) for 12 hours, but, in this case, the HSP70 level was very similar to that noted in freshly prepared fragments at the start of incubation (Figure $5 \mathrm{~b}$ ). In contrast to the effects noted with antisense oligonucleotide, incubation of fragments with sense oligonucleotide (S I or S II) did not reduce the rise in HSP70 levels noted after 12 hours of culture. HSC70 and HSP27 levels were also unaffected by treatment with AS or sense oligonucleotides (Figure 5b). A similar trend in HSP70 mRNA expression was observed when the fragments were incubated with antisense or sense oligonucleotides (data not shown). Neither antisense nor sense oligonucleotide addition altered HSP60 mRNA expression in fragments after 12 hours of culture (data not shown). Effects of inbibiting HSP70 expression on colocalization, trypsinogen activation, and cell injury in pancreas fragments. As shown in Figure 6, supramaximal stimulation with cerulein of fragments cultured with quercetin for 12 hours results in a rise in intracellular trypsin activity, but no such rise is noted when quercetin is absent from the culture medium. Similarly, supramaximal stimulation with cerulein of fragments cultured with antisense nucleotides to HSP70 results in a rise in trypsin activity and TAP levels in the fragments, but no such rise is noted when sense oligonucleotides are used (Figure 7a and b). Finally, supramaximal stimulation with cerulein of fragments cultured with an antisense oligonucleotide (AS I or AS II) results in accelerated $\mathrm{LDH}$ release, but no such acceleration is present when a sense oligonucleotide (S I or S II) is used (Figure 7c). As previously noted to occur in vivo (4), in vitro exposure of freshly prepared fragments to a supramaximally stimulating dose of cerulein for 30 minutes results 

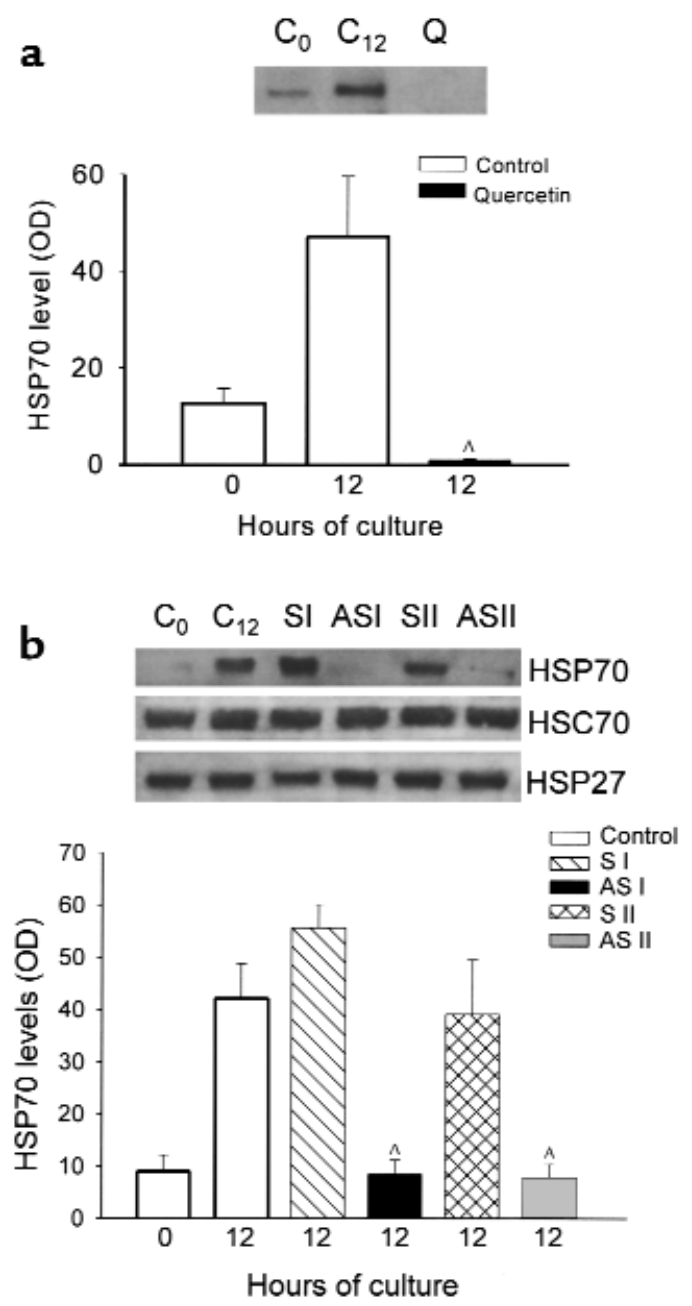

Figure 5

Effect of incubation of pancreas fragments with quercetin or antisense/sense HSP70 oligonucleotides on HSP expression. (a) $\mathrm{C}_{0}$, freshly prepared pancreas fragments; $C_{12}$, pancreas fragments after 12 hours of culture; $\mathrm{Q}$, pancreas fragments after 12 hours of culture with quercetin $(50 \mu \mathrm{M})$. HSP70 protein expression was evaluated by Western blotting, and relative optical densities are expressed as mean \pm SEM for at least three separate experiments in each group. ${ }^{A} P<0.01$ when quercetin-incubated fragments are compared with untreated 12-hour control fragments. (b) $C_{0}$, freshly prepared pancreas fragments; $C_{12}$, pancreas fragments after 12 hours of culture; $\mathrm{SI}$, pancreas fragments incubated with sense oligonucleotide $\mathrm{S} I(1 \mu \mathrm{M})$ for 12 hours; AS I, pancreas fragments incubated with 14-mer antisense oligonucleotide AS I ( $1 \mu \mathrm{M})$ for 12 hours; S II, pancreas fragments incubated with sense oligonucleotide S II (1 $\mu \mathrm{M})$ for 12 hours; AS II, pancreas fragments incubated with 18-mer antisense oligonucleotide AS II $(1 \mu \mathrm{M})$ for 12 hours. Expression of indicated HSPs was assessed by Western blotting. Relative optical densities of HSP70 bands are expressed as mean \pm SEM for at least three separate experiments in each group. ${ }^{A} P<0.01$ when antisense oligonucleotide-incubated fragments are compared with untreated 12-hour control fragments.

in colocalization of cathepsin B and TAP (Figure 8a). This colocalization is prevented when fragments are cultured for 12 hours (Figure 8b). Incubation of fragments with AS-HSP70 oligonucleotide (AS I) results in restoration of this colocalization phenomenon in response to supramaximal cerulein (Figure 8c).

\section{Discussion}

HSPs are a group of proteins that are preferentially expressed in many cell types in response to stress. For the most part, HSPs exert a protective effect against cellular injury $(22,23)$. HSP expression can be induced by a variety of physiological stresses including heat, oxygenderived free radicals, endotoxins, heavy metal ions, amino acids, ethanol, and arsenite (24). HSP70 (also referred to as HSP72 or inducible HSP) is perhaps the most well-studied group of cytoprotective HSPs (25). Members of the HSP70 family are believed to act as molecular chaperones accompanying newly synthesized proteins through various intracellular compartments, facilitating passage of proteins across various intracellular barriers, maintaining proper folding of newly synthesized proteins, and aiding in the elimination of incorrectly synthesized or assembled proteins $(26,27)$. In general, stressed cells initially translate HSP messages to the exclusion of other messages (28). This preferential translation appears to reflect both transcriptional (29, 30 ) and posttranscriptional (31) regulatory mechanisms.

In previous in vitro studies, we have employed a model system comprising freshly prepared acini obtained after collagenase digestion and gentle shearing of pancreatic tissue $(4,5)$. Preparation of acini, however, has been noted previously to result in spontaneous stress-activated protein kinase activation, chemokine and cytokine upregulation, and HSP27 phosphorylation $(32,33)$. Presumably, these changes represent cellular responses to the stress of acini preparation. To avoid the enzymatic digestion and shearing that could contribute to acinar cell stress, we have employed in the current study a model system consisting of pancreas fragments rather than acini. Even under these conditions, however, we have found that HSP70 expression increases rapidly during culture. Both HSP70 mRNA and HSP70 protein levels are increased. Two potential explanations for our observations should be considered. First is the possibility that simple in vitro incubation of the fragments represents a stress that is sufficient to increase HSP70 expression. Alternatively, our observations could indicate that the in vitro condition results in the elimination of a tonically inhibitory influence that physiologically prevents HSP70 expression in vivo.

Pancreas fragments, cultured for short periods, have been used by others, most notably Norman and coworkers (13), for studies dealing with pancreatitis. We have found that those fragments remain viable for up to 48 hours (Figure 1a) of culture and that they retain the normal biphasic dose-dependent amylase secretory response to increasing concentrations of cerulein for up to 24 hours (Figure 2). This latter observation indicates that the affinity states of the CCK-A receptor on pancreatic acinar cells, which normally involve high-affinity stimulatory receptors and lower-affinity inhibitory receptors, are not altered during these relatively brief periods of culture.

Freshly prepared fragments of pancreas respond to supramaximal secretagogue stimulation in a manner 


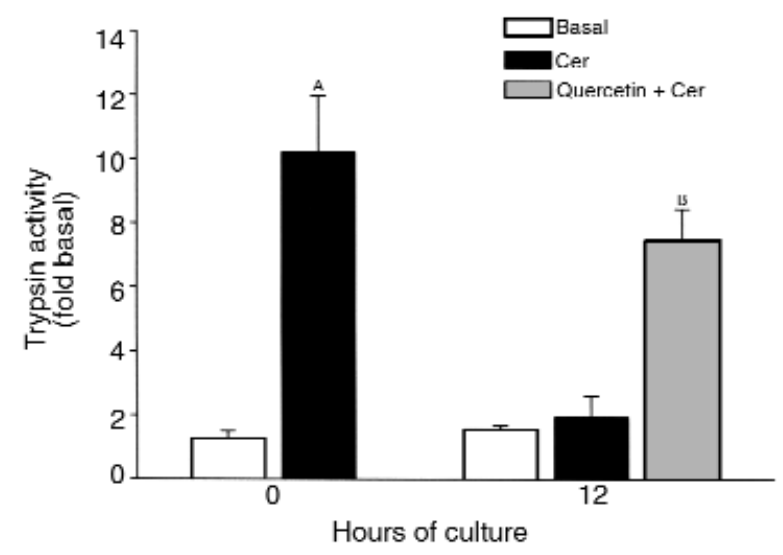

Figure 6

Effect of incubation of pancreas fragments with quercetin $(50 \mu \mathrm{M})$ on trypsinogen activation. Trypsin activity in the homogenates was measured according to the method described in the text. Values are mean \pm SEM obtained from at least three separate experiments. ${ }^{A} P<0.05$ when cerulein-treated freshly prepared fragment values are compared with basal values. ${ }^{B} P<0.05$ when quercetinincubated cerulein-stimulated fragments are compared with cerulein-stimulated 12-hour control fragments.

that is identical to that noted for freshly prepared acini and similar to that noted for the in vivo pancreas after supramaximal cerulein stimulation; i.e., there is colocalization of lysosomal hydrolases and digestive enzyme zymogens, intracellular activation of trypsinogen, and subsequent acinar cell injury. Evidence for the trypsinogen activation comes from the observation that TAP levels and trypsin activity in the fragments are increased after addition of cerulein, whereas evidence for the acinar cell injury comes from the finding that $\mathrm{LDH}$ release from fragments is accelerated after cerulein addition (Figure 3).

Within 6 hours after the start of culture, the ability of a supramaximally stimulating concentration of cerulein to increase TAP or trypsin activity levels or to cause accelerated LDH release from fragments is lost (Figure 3), whereas HSP70 levels are markedly increased by 6 hours after the start of culture (Figure 4).

The similarity in the time dependence for HSP70 expression and loss of response to supramaximal secretagogue stimulation suggested to us that the two phenomena might be related and that HSP70 might protect against cerulein-induced cell injury by preventing trypsinogen activation. To further explore this possibility, we have employed two methods of inhibiting HSP70 expression - quercetin and antisense oligonucleotide addition to the incubation medium - and evaluated the effects of these agents on the cerulein-induced changes.

Quercetin and several other flavonoids have been used extensively to prevent the expression of HSP70 in several models. Quercetin has been shown to inhibit HSP70 expression by blocking binding of heat shock factor (HSF) to heat shock element (HSE) and to reduce HSP70 mRNA accumulation $(34,35)$. In our experiments, inclusion of quercetin in the culture medium for 12 hours prevented culture-induced upregulation of HSP70 expression (Figure 5a) and restored the ability of a supramaximally stimulating concentration of cerulein to cause intracellular trypsinogen activation (Figure 6).

These observations are clearly consistent with the concept that the culture-dependent loss of trypsinogen activation in response to cerulein is mediated by the culture-dependent increase in HSP70 expression.
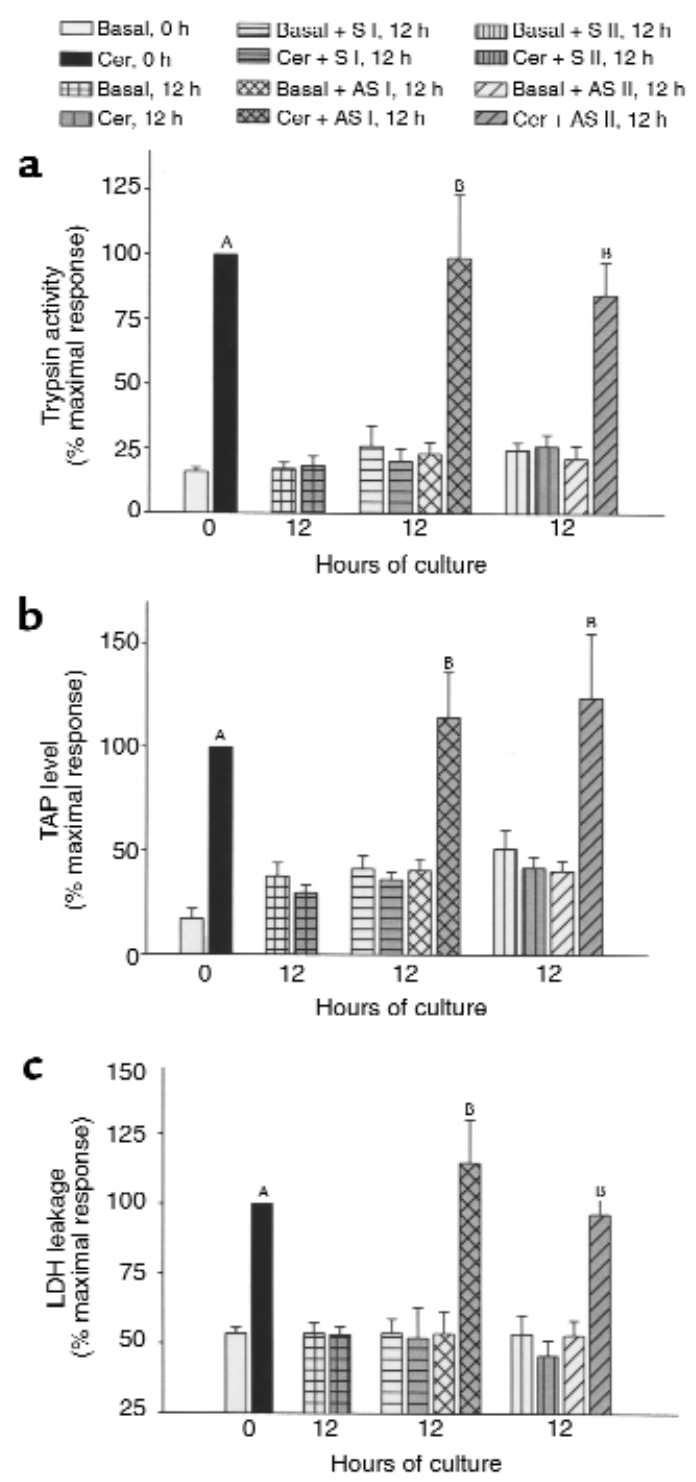

Figure 7

Effect of incubation of pancreas fragments with antisense/sense HSP70 oligonucleotides $(1 \mu \mathrm{M})$ on trypsinogen activation and cell injury as assessed by LDH release into the medium. Trypsin activity (a), TAP levels (b), and LDH leakage (c) were measured as described in text. Values are mean \pm SEM obtained from at least three independent experiments and are expressed as percent of maximal response to cerulein stimulation in freshly prepared pancreas fragments. ${ }^{A} P<0.05$ when cerulein-treated freshly prepared fragment values are compared with the basal values. ${ }^{B} P<0.05$ when antisense oligonucleotide-incubated cerulein-stimulated fragments are compared with cerulein-stimulated 12-hour control fragments. 

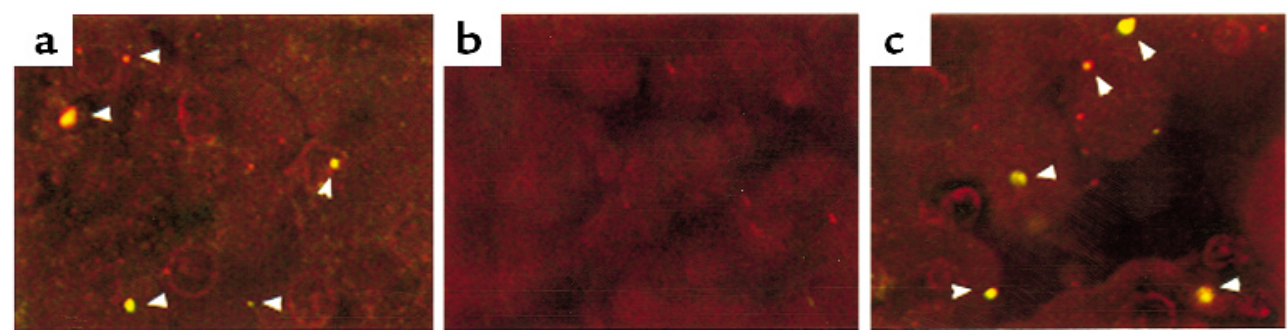

\section{Figure 8}

Light microscopic colocalization of TAP and cathepsin B in rat pancreas fragments, 30 minutes after stimulation with $0.1 \mu \mathrm{M}$ cerulein. (a) Freshly prepared fragments. (b) Fragments after 12 hours of culture. (c) Fragments incubated with AS I nucleotide for 12 hours. Doublelabeling fluorescence microscopy of paraffin-embedded sections was carried out with TAP antibody/FITC-conjugated anti-rabbit IgG and anti-cathepsin B/rhodamine-conjugated anti-goat IgG. The areas positive for both TAP and cathepsin B appear as yellow dots. Arrowheads in $\mathbf{a}$ and $\mathbf{c}$ indicate the areas positive for both cathepsin B and TAP. The images shown are representative of several examined in each group in three independent experiments.

Unfortunately, however, other conclusions also remain possible since quercetin has a number of other effects on cells in addition to inhibiting HSP expression, and even the effects on HSP expression are nonspecific for HSP70. Studies by others using nonpancreatic tissues have shown that quercetin inhibits synthesis of HSP110, HSP90, HSP47, HSP40, and HSP28, in addition to HSP70 (34).

To examine the role of HSP70 more specifically in cerulein-induced pancreatic injury, we have chosen to employ antisense HSP70 oligonucleotides. Theoretically, introduction of an antisense oligonucleotide, which is complementary to a portion of the coding sequence, will block expression of the protein encoded by the target mRNA. Recently, stable analogues of antisense nucleotides incorporating phosphorothioates have become available and have been extensively evaluated $(36,37)$. Nakano et al. (8) used antisense HSP70 to block hypoxia and ischemia-induced HSP70 increases in feline cardiocytes. They showed that inhibition of HSP70 expression in those cells increases their susceptibility to hypoxic injury. Guzik et al. (38) blocked heat-induced HSP70 expression in monocytes using an antisense oligomer to show that HSP70 protected monocytes against Staphylococcus aureus-induced apoptosis. In our studies, we have used phosphorothioate antisense HSP70 to inhibit HSP70 expression induced by culture of pancreas fragments. We have compared the effects noted with the antisense HSP70 oligonucleotide with that observed when either no oligonucleotide is added or when the sense HSP70 oligonucleotide is present.

Addition of either sense or antisense oligonucleotide to the culture medium does not alter the viability of cultured fragments, as evaluated using the MTT assay, for up to 12 hours (Figure 1a). Addition of the antisense oligonucleotide (AS I or AS II), but not the sense (S I or S II) oligonucleotide, prevents both HSP70 mRNA and HSP70 protein expression after 12 hours of culture. These findings are consistent with the conclusion that the antisense HSP70 oligonucleotide blocks HSP70 expression. The effect appears to be specific for
HSP70 since HSC70 and HSP27 protein levels and HSP60 mRNA expression are not altered by incubation with the antisense oligonucleotide.

The ultimate goal of our studies was to evaluate the possible cause-effect relationship between cultureinduced HSP70 expression in pancreas fragments and culture-induced protection against both ceruleininduced trypsinogen activation and cerulein-induced cell injury. To examine these issues, fragments were cultured in the presence of either antisense or sense oligonucleotides for 12 hours and then subjected to supramaximal secretagogue stimulation. As shown in Figures $7, a-c$, and Figure 8, fragments incubated in the absence of oligonucleotides did not demonstrate colocalization, TAP generation, trypsin activity, or accelerated LDH leakage after addition of cerulein. A similar lack of response was noted when the sense oligonucleotide was added to the incubation medium. However, when the antisense oligonucleotide was present at a concentration that prevented HSP70 expression, a marked increase in colocalization, TAP level, trypsin activity, and LDH release were observed after addition of cerulein.

The restoration of these responses by inclusion of a specific probe (i.e., antisense HSP oligonucleotide) allows us to conclude, unequivocally, that the loss of these responses induced by culture is mediated by the culture-induced rise in HSP70 expression. Since TAP is released from trypsinogen during zymogen activation, our finding that HSP70 prevents both the release of TAP and the rise in trypsin activity in fragments indicates that the HSP exerts its effect by blocking the intracellular activation of trypsinogen rather than inhibiting the already activated enzyme.

Prior thermal stress protects against cerulein-induced pancreatitis (8) and prevents cerulein-induced in vivo activation of trypsinogen in acinar cells (39). Whereas these phenomena are associated with thermal stress-induced HSP70 expression, thermal stress may have other effects, including induction of other HSPs, which could account for protection against pancreatitis and prevention of trypsinogen activation in vivo. The current reported results clearly indicate that HSP70 
expression can prevent intra-acinar cell activation of trypsinogen and cell injury caused by cerulein in vitro when that HSP70 expression is induced by culture stress. Further studies using the in vivo system, however, will be needed before it can be unequivocally concluded that the protection afforded by thermal stress is actually mediated by HSP 70. Furthermore, the mechanism(s) by which HSP70 might interfere with cerulein-stimulated intracellular trypsinogen activation also remain unknown and will be the subject of future studies. That process of intracellular activation is believed by some, but not all, investigators to result from missorting of proteins within acinar cells and the resultant colocalization of trypsinogen and other digestive enzymes' zymogens with lysosomal hydrolases such as cathepsin B within membrane-bound intracellular organelles (40). The observations reported in this study, along with the recent reports of studies in various systems that suggest that HSP70 may play an important role in intracellular trafficking of proteins $(41,42)$, makes it tempting to speculate that HSP70 might prevent cerulein-induced intracellular activation of trypsinogen by interfering with the cerulein-induced colocalization phenomenon that we have shown in here (Figure 8).

\section{Acknowledgments}

The authors thank J. Norman and C. Jaffrey for providing the protocol for preparation of pancreas fragments, and Uma Akella and Amy Pan for expert technical assistance. The authors are also grateful to Dr. Lalit C. Garg, National Institute of Immunology, Delhi, India, for his helpful suggestions. This work was supported in part by NIH DK-31396.

1. Lampel, M., and Kern, H.F. 1977. Acute interstitial pancreatitis in rats induced by excessive doses of a pancreatic secretagogue. Virchows Arch. A. Pathol. Anat. Histol. 373:97-113.

2. Grady, T., Saluja, A., Kaiser, A., and Steer, M. 1996. Edema and intrapancreatic trypsinogen activation precede glutathione depletion during caerulein pancreatitis. Am. J. Physiol. 271:G20-G26.

3. Saluja, A.K., et al. 1997. Caerulein-induced in vitro activation of trypsinogen in rat pancreatic acini is mediated by cathepsin B. Gastroenterology. 113:304-310.

4. Hofbauer, B., et al. 1998. Intra-acinar activation of trypsinogen during caerulein-induced pancreatitis in rats. Am. J. Physiol. 275:G352-G362.

5. Saluja, A.K., et al. 1999. Secretagogue-induced digestive enzyme activation and cell injury in pancreatic acini. Am. J. Physiol. 276:G835-G842.

6. Wagner, A.C., et al. 1996. Hyperthermia induces heat shock protein expression and protection against cerulein-induced pancreatitis in rats. Gastroenterology. 111:1333-1342.

7. Schafer, C., and Williams, J.A. 2000. Stress kinases and heat shock proteins in the pancreas: possible roles in normal function and disease. J. Gastroenterol. 35:1-9.

8. Nakano, M., Mann, D.L., and Knowlton, A.A. 1997. Blocking the endogenous increase in HSP72 increases susceptibility to hypoxia and reoxygenation in isolated adult feline cardiocytes. Circulation. 95:1523-1531.

9. Hunt, C., and Morimoto, R.I. 1985. Conserved features of eukaryotic hsp70 genes revealed by comparison with the nucleotide sequence of human hsp70. Proc. Natl. Acad. Sci. USA. 82:6455-6459.

10. Dworniczak, N., and Mirault, M.-E. 1987. Structure and expression of a human gene encoding for a $71 \mathrm{kd}$ heat shock 'cognate' protein. Nucleic Acids Res. 15:5181-5197.

11. Hunt, C., and Calderwood, S. 1990. Characterization and sequence of a mouse hsp70 gene and its expression in mouse cell lines. Gene. 87:199-204.

12. Robertson, J.D., Datta, K., Biswal, S.S., and Kehrer, J.P. 1999. Heat-shock protein 70 antisense oligomers enhance proteasome inhibitor-induced apoptosis. Biochem. J. 344:477-485.
13. Jaffrey, C., Eichenbaum, D., Denham, D.W., and Norman, J. 1999. A novel pancreatic model: the snip method of pancreatic isolation for in vitro study. Pancreas. 19:377-381.

14. Bradford, M.M. 1976. A refined and sensitive method for the quantitation of microgram quantities of protein utilizing the principle of protein-dye binding. Anal. Biochem. 72:248-254.

15. Laemmli, U.K. 1970. Cleavage of structural proteins during the assembly of the head of bacteriophage $\mathrm{T}_{4}$. Nature. 227:680-685.

16. Towbin, H., Staehelin, T., and Gordon, J. 1979. Electrophoretic transfer of proteins from polyacrylamide gels to nitrocellulose sheets: procedure and some applications. Proc. Natl. Acad. Sci. USA. 76:4350-4354.

17. Kruse-Jarres, J.D., et al. 1989. Evaluation of a new alpha-amylase assay using 4.6-ethylidene-(G7)-1-4-nitrophenyl-(G1)-alpha-D-maltoheptaoside as substrate. J. Clin. Chem. Clin. Biochem. 27:103-113.

18. Kawabata, S., et al. 1988. Highly sensitive peptide-4-methylcouramylamide substrates for blood clotting proteases and trypsin. Eur. J. Biochem. 172:17-25.

19. Labarca, C., and Paigen, K. 1980. A simple, rapid and sensitive DNA assay procedure. Anal. Biochem. 102:344-352.

20. Gudgeon, A.M., et al. 1990. Trypsinogen activation peptide assay in the early prediction of severity of acute pancreatitis. Lancet. 335:4-8.

21. Amador, E., Dorman, L.E., and Wacker, W.E.C. 1936. Serum lactic dehydrogenase: an analytical assessment of current assays. Clin. Chem. 9:391.

22. Welch, W.J. 1992. Mammalian stress response: cell physiology, structure/function of stress proteins, and implications for medicine and disease. Physiol. Rev. 72:1063-1081.

23. Williams, R.S., Thomas, J.A., Fina, M., German, Z., and Benjamin, I.J. 1993. Human heat shock protein 70 (hsp70) protects murine cells from injury during metabolic stress. J. Clin. Invest. 92:503-508.

24. Wong, H.R., and Wispé, J.R. 1997. The stress response and the lung. Am.J. Physiol. 273:L1-L9.

25. Kiang, J.G., and Tsokos, G.C. 1998. Heat shock protein 70 kDa: molecular biology, biochemistry and physiology. Pharmacol. Ther. 80:183-201.

26. Mathew, A., and Morimoto, R.I. 1998. Role of the heat-shock response in the life and death of proteins. Ann. NY Acad. Sci. 851:99-111.

27. Benjamin, I.J., and McMillan, D.R. 1998. Stress (heat shock) proteins. Molecular chaperones in cardiovascular biology and disease. Circ. Res. 83:117-132

28. Mizzen, L.A., and Welch, W.J. 1988. Characterization of the thermotolerant cell. I. Effects on protein synthesis activity and the regulation of heatshock protein 70 expression. J. Cell. Biol. 106:1105-1116.

29. Wu, B., Hunt, C., and Morimoto, R. 1985. Structure and expression of the human gene encoding major heat shock protein HSP70. Mol. Cell. Biol. 5:330-341.

30. Kingston, R.E., Schuetz, T.J., and Larin, Z. 1987. Heat-inducible human factor that binds to a human hsp70 promoter. Mol. Cell. Biol. 7:1530-1534.

31. Beckmann, R.P., Mizzen, L.E., and Welch, W.J. 1990. Interaction of Hsp 70 with newly synthesized proteins: implications for protein folding and assembly. Science. 248:850-854.

32. Grady, T., Dabrowski, A., Williams, J.A., and Logsdon, C.D. 1996. Stressactivated protein kinase activation is the earliest direct correlate to the induction of secretagogue-induced pancreatitis in rats. Biochem. Biophys. Res. Commun. 227:1-7.

33. Groblewski, G.E., et al. 1997. Cholecystokinin stimulates heat shock protein 27 phosphorylation in rat pancreas both in vivo and in vitro. Gastroenterology. 112:1354-1361.

34. Hosokawa, N., et al. 1990. Flavonoids inhibit the expression of heat shock proteins. Cell Struct. Funct. 15:393-401.

35. Hosokawa, N., et al. 1992. Inhibition of the activation of heat shock factor in vivo and in vitro by flavonoids. Mol. Cell. Biol. 12:3490-3498.

36. Agrawal, S. 1996. Antisense oligonucleotides: towards clinical trials. Trends Biotechnol. 14:378-387.

37. Agrawal, S., and Zhao, Q. 1998. Antisense therapeutics. Curr. Opin. Chem. Biol. 2:519-528.

38. Guzik, K., Bzowska, M., Dobrucki, J., and Pryjma, J. 1999. Heat-shocked monocytes are resistant to Staphylococcus aureus-induced apoptotic DNA fragmentation due to expression of HSP72. Infect. Immun. 67:4216-4222.

39. Frossard, J.L., et al. 1998. Heat shock protein (HSP) 70 expression reduces the severity of caerulein-induced acute pancreatitis by reducing intrapancreatic active trypsin levels. Gastroenterology. 114:G1868. (Abstr.)

40. Steer, M.L., and Saluja, A.K. 1993. Experimental acute pancreatitis: studies of the early events that lead to cell injury. In The pancreas: biology, pathobiology and disease. 2nd edition. V.L.W. Go et al., editors. Raven Press. New York, New York, USA. 489-499.

41. Pilon, M., and Schekman, R. 1999. Protein translocation: how Hsp70 pulls it off. Cell. 97:679-682.

42. Velez-Granell, C.S., Arias, A.E., Torres-Ruiz, J.A., and Bendayan, M. 1994. Molecular chaperones in pancreatic tissue: the presence of cpn10, cpn60 and hsp70 in distinct compartments along the secretory pathway of the acinar cells. J. Cell. Sci. 107:539-549. 